\title{
POWERS OF A MATRIX OVER A LATTICE
}

W. R. UTZ

Let $M=\left\|a_{i j}\right\|$ be an $n \times n$ matrix with elements in a lattice in which meet, join, and inclusion are denoted by $a b, a+b$, and $a \subset b$, respectively. Two $n \times n$ lattice matrices $M$ and $M^{\prime}$ are said to be equal if $a_{i j}=a_{i j}^{\prime}, i, j=1,2, \cdots, n$, and if $a_{i j} \subset a_{i j}^{\prime}, i, j=1,2, \cdots, n$, then we shall write $M \subset M^{\prime}$. The $n^{2}$ elements of $M$ will be called independent provided $P_{1} \subset \sum_{k=2}^{r} P_{k}$, where $P_{1}, P_{2}, \cdots, P_{r}$ are any products of elements $a_{i j}$ of $M$, implies $P_{1} \subset P_{m}$ for some $m$ such that $2 \leqq m \leqq r$.

THEOREM 1. If the elements of $M$ are in a distributive lattice, the sequence $M, M^{2}, M^{3}, \ldots$ of powers of $M$ under ordinary matrix multiplication contains at most $(n-1)^{2}+N$ distinct matrices where $N$ is the least common multiple of $1,2, \cdots, n$. At most $N$ of these matrices occur periodically in the sequence. If the elements of $M$ are independent, there are exactly $(n-1)^{2}+N$ distinct matrices in the sequence of which $N$ occur periodically.

The proof of this theorem follows very closely that of the corresponding theorem given by Sanders ${ }^{1}$ for powers of a matrix with elements as sets of points. Because of this similarity the proof will not be given here.

It may be of interest to observe that in case the elements of $M$ are independent, then the collection

$$
M^{s+1}, M^{s+2}, \cdots, M^{s+N} \quad\left(s=(n-1)^{2}\right),
$$

form a cyclic group of order $N$ under matrix multiplication with $M^{N}$ as identity.

The following theorem holds without distribution in the lattice.

THEOREM 2. If $M=\left\|a_{i j}\right\|$ is an $n \times n$ matrix over a lattice and if $a_{i j} \subset a_{m p}$ when $i \geqq m$ and $j \geqq p$, then $M \subset M^{2}=M^{w}$ for all integers $w \geqq 2$.

Proof. Let $a_{i j}^{(2)}=\sum_{s=1}^{n} a_{i s} a_{s j}$ denote the element in the $i$ th row and $j$ th column of $M^{2}$. Since $\left(a_{i 1} a_{1 j}\right)\left(a_{i s} a_{s j}\right)=\left(a_{i 1} a_{i s}\right)\left(a_{1 j} a_{s j}\right)=a_{i s} a_{s j}$ for $s=1,2, \cdots, n$, we have $a_{i 1} a_{1 j}+a_{i s} a_{s j}=a_{i 1} a_{1 j}$ and $a_{i j}^{(2)}=a_{i 1} a_{1 j}$. Thus $a_{i j} a_{i j}^{(2)}=a_{i j} a_{i 1} a_{1 j}=a_{i j}$ hence $a_{i j} \subset a_{i j}^{(2)}$ and $M \subset M^{2}$.

Presented to the Society, November 25, 1950; received by the editors April 15, 1950.

${ }^{1}$ A linear transformation whose variables and coefficients are sets of points, Bull. Amer. Math. Soc. vol. 48 (1942) pp. 440-447. 
If $i \geqq m$ and $j \geqq p$, then $\left(a_{i 1} a_{1 j}\right)\left(a_{m 1} a_{1 p}\right)=\left(a_{i 1} a_{m 1}\right)\left(a_{1 j} a_{1 p}\right)=a_{i 1} a_{1 j}$, hence $a_{i j}^{(2)} \subset a_{m p}^{(2)}$.

To show that $M^{w}=M^{2}$ for $w>2$, it is sufficient to show that $M^{3}=M^{2}$. Let $a_{i j}^{(3)}=\sum_{s=1}^{n} a_{i s} a_{s j}^{(2)}$ denote the element in the $i$ th row and $j$ th column of $M^{3}$. As before, $\left(a_{i 1} a_{1 j}^{(2)}\right)\left(a_{i s} a_{s j}^{(2)}\right)=\left(a_{i 1} a_{i s}\right)\left(a_{1 j}^{(2)} a_{s j}^{(2)}\right)$ $=a_{i s} a_{s j}^{(2)}$ for $s=1,2, \cdots, n$, hence $a_{i 1} a_{1 j}^{(2)}+a_{i s} a_{s j}^{(2)}=a_{i 1} a_{1 j}^{(2)}$ and $a_{i j}^{(3)}$ $=a_{i 1} a_{i j}^{(2)}=a_{i 1} a_{11} a_{1 j}=a_{i 1} a_{1 j}=a_{i j}^{(2)}$. This completes the proof of the theorem.

Theorem 2 holds if the inequalities are reversed, that is, if $a_{i j} \subset a_{m p}$ when $i \leqq m$ and $j \leqq p$. From the proof of the preceding theorem one infers that $M=M^{2}$ if, and only if, $a_{i 1} a_{1 j}=a_{i j}$ for all $i, j$.

As can be easily seen, any $n \times n$ lattice matrix $\left\|a_{i j}\right\|$ can be reduced by a sequence of additions of one row or column to another to a matrix with general element

$$
c_{i j}=\sum_{r=i}^{n} \sum_{r=j}^{n} a_{r s}
$$

In this matrix $c_{i j} \subset c_{m p}$ if $i \geqq m$ and $j \geqq p$.

UNIVERSITY OF MISSOURI 